\title{
Monolithic diamond Raman laser
}

\author{
Sean Reilly*, Vasili G Savitski, Hangyu Liu, Erdan Gu, Martin D Dawson, and Alan J Kemp \\ Institute of Photonics, Department of Physics, SUPA, University of Strathclyde, 106 Rottenrow, Glasgow G4 ONW, UK \\ *Corresponding author: s.reilly@strath.ac.uk
}

Received Month X, XXXX; revised Month X, XXXX; accepted Month X,

XXXX; posted Month X, XXXX (Doc. ID XXXXX); published Month X, XXXX

\begin{abstract}
A monolithic diamond Raman laser is reported. It utilizes a $13 \mathrm{~mm}$ radius of curvature lens etched onto the diamond surface and dielectric mirror coatings to form a stable resonator. The performance is compared to that of a monolithic diamond Raman laser operating in a plane-plane cavity. On pumping with a compact Q-switched laser at $532 \mathrm{~nm}(16 \mu \mathrm{J}$ pulse energy; $1.5 \mathrm{~ns}$ pulse duration; $10 \mathrm{kHz}$ repetition-rate; $\mathrm{M}^{2}<1.5$ ), laser action was observed at the first, second and third Stokes wavelengths $(573 \mathrm{~nm}, 620 \mathrm{~nm}$ and $676 \mathrm{~nm}$ respectively) in both cases. For the microlens cavity, a conversion efficiency of $84 \%$ was achieved from the pump to the total output power, with a slope efficiency of $88 \%$. This compares to a conversion efficiency of $59 \%$, and a slope efficiency of $74 \%$ for the plane-plane case. Total Raman output powers of $134 \mathrm{~mW}$ and $96 \mathrm{~mW}$ were achieved for the micro-lens and plane-plane cavities respectively.
\end{abstract}

Recent advances in diamond growth [1] have allowed laser engineers to exploit the material's unrivalled thermo-mechanical properties and large Raman gain coefficient [2]. This has enabled, for example, demonstrations of high average power continuous wave [3], [4] and pulsed [5]-[7] diamond Raman lasers at hard to reach, but commercially significant, wavelengths from the ultraviolet to the mid-infrared [8], [9]. The yelloworange spectral region is of particular interest, with applications in ophthalmology [10], [11]; however there are few simple, robust and compact yellow orange lasers available.

In this letter, the feasibility of compact and robust monolithic diamond Raman lasers is demonstrated and the performance characteristics are reported. A comparison is drawn between a $2 \mathrm{~mm}$ long device that uses a microlens structure to form a stable cavity and a $1.6 \mathrm{~mm}$ long plane-plane resonator. Conversion efficiencies of $84 \%$ and $59 \%$, respectively, are achieved despite modest pump pulse energies of $<20 \mu \mathrm{J}$. These efficiencies are comparable to the $64 \%$ reported in a conventional diamond Raman laser pumped at millijoule levels [12].

The single crystal synthetic diamond used to achieve these results was grown using chemical vapour deposition by Element $6 \mathrm{Ltd}$. Its birefringence was $<1 \times 10^{-6}$ and it had a specified absorption coefficient of $<0.005 \mathrm{~cm}^{-1}$ at $1064 \mathrm{~nm}$. The sample used for the plane-plane resonator was $3.6 \times 2 \times 1.6 \mathrm{~mm}^{3}$, while that used for the micro-lens cavity was $4 \times 4 \times 2 \mathrm{~mm}^{3}$.

Microlens structures were created on the latter sample using a multi-layer "resist-reflow" process, based on [13], followed by an inductively coupled plasma etch using $\mathrm{Ar} / \mathrm{Cl}_{2}$. An $\mathrm{Ar} / \mathrm{Cl}_{2}$ plasma etch was chosen over more conventional $\mathrm{Ar} / \mathrm{O}_{2}$ recipes because the low etch selectivity between diamond and photoresist enabled the fabrication of micro-lenses with a larger radius of curvature. An array of $8 \times 8$ spherical micro-lenses with a diameter of approximately $400 \mu \mathrm{m}$ and radii of curvature of around $13 \mathrm{~mm}$ were etched on one of the $4 \times 4 \mathrm{~mm}^{2}$ surfaces. This is a relatively long radius of curvature for a microlens fabricated by resist-reflow on diamond, and was chosen to provide the largest possible cavity mode area, hence reducing the risk of coating damage in the microlens sample.

The back surfaces of both diamonds were coated for high-reflection (HR) at 532nm, to double pass the pump, and partial reflection (PR) at the first Raman shifted wavelength of $573 \mathrm{~nm}(70 \%$ reflectivity). The front surfaces were coated for high-transmission (HT) at the pump wavelength $(\sim 20 \%$ reflectivity) and $\mathrm{HR}$ coated at $573 \mathrm{~nm}$.

These coated samples were tested in the experimental setup shown in Figure 1 and Figure 2. Both were pumped with an Elforlight SPOT laser emitting Q-switched pulses at $532 \mathrm{~nm}$ with pulse durations of $1.5 \mathrm{~ns}$ (full width at half

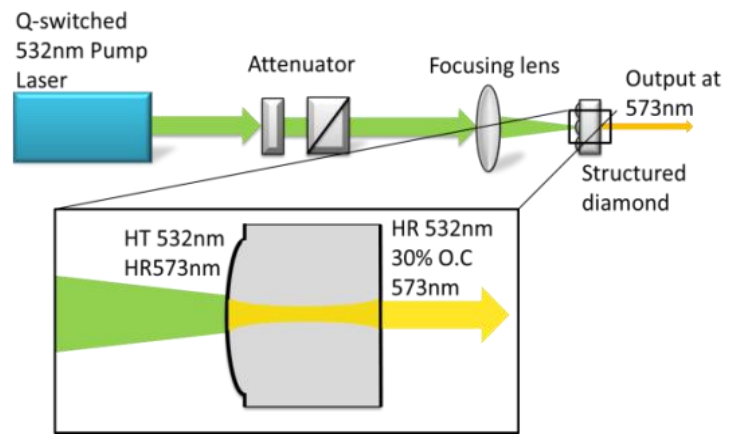

Figure 1. Experimental setup used to achieve Raman laser action in microlens and plane-plane diamond. HT: high transmission; HR: high reflectivity; O.C.: output coupling.

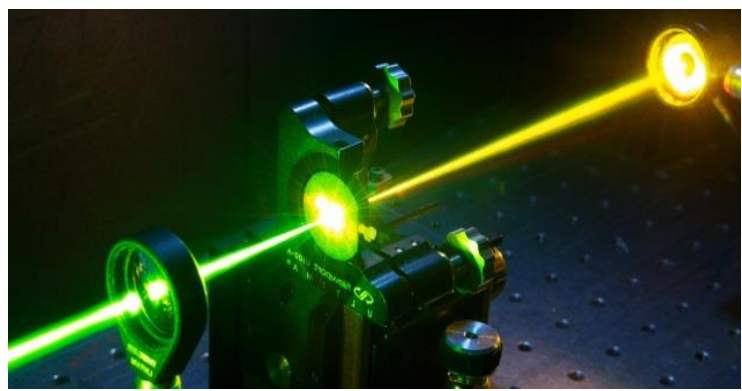

Figure 2. Green to yellow/orange conversion using a monolithic diamond Raman resonator. The photograph was taken at maximum pump power with all three Stokes orders present. 
maximum, FWHM) and pulse repetition rates between $1 \mathrm{kHz}$ and $10 \mathrm{kHz}$. The results presented in this paper were all taken at $10 \mathrm{kHz}$; however, comparable conversion efficiencies were observed at $1 \mathrm{kHz}$.

The pump was attenuated using a combination of a half wave-plate and a polarizing cube. It was then focused using a $50 \mathrm{~mm}$ focal length lens. In the case of the microlens sample, it was focused through a single microlens structure onto the back surface of the diamond. This resulted in a pump spot radius of $9 \mu \mathrm{m}$, which was found to provide slightly better performance than a pump spot size of $18 \mu \mathrm{m}$. The microlens cavity had a fundamental mode radius of $24 \mu \mathrm{m}$. Pump light propagated along a $<110>$ direction in the diamond, and was polarized along a $<100>$ direction in the micro-lens case, whilst in the plane-plane system, pump light propagated along a $<100>$ direction and was polarized along a $<110>$ direction.

This set-up resulted in Raman conversion of the green pump at $532 \mathrm{~nm}$ to the yellow at $573 \mathrm{~nm}$ when the pump pulse energy reached $1.5 \mu \mathrm{J}$ for the micro-lens case and 3.7 $\mu \mathrm{J}$ for the plane-plane case. The pump energies quoted are corrected for the $20 \%$ reflectivity of the front coating on both diamonds. The reflectivities at the $2^{\text {nd }}$ Stokes wavelength $(620 \mathrm{~nm})$ of the coatings deposited on the back and front surfaces of the diamonds were $50 \%$ and $40 \%$, respectively. Even with this relatively high combined output coupling of $80 \%$ per round trip, a significant fraction of the pump power was converted to the $2^{\text {nd }}$ Stokes wavelength: $59 \%$ and $25 \%$, respectively, for the microlens and plane-plane cases at maximum pump power (blue triangles in Figure 3a and Figure 3b). A small amount of $3^{\text {rd }}$ Stokes emission was also present as the pump pulse energy reached maximum (red circles in Figure $3 \mathrm{a}$ and Figure 3b). Clamping of the $1^{\text {st }}$ Stokes (green squares in Figure 3a) is observed for the microlens resonator when the $2^{\text {nd }}$ Stokes rises above threshold, but not in the plane-plane case (green squares in Figure 3b). In the case of the microlens cavity, the pre-defined cavity mode confines the $1^{\text {st }}$ Stokes, allowing the $2^{\text {nd }}$ Stokes to efficiently extract energy. In the plane-plane cavity, however, the cavity mode is less restricted, hence potentially less conducive to efficient $2^{\text {nd }}$ Stokes generation. These power transfer curves were measured using a set of calibrated filters. The output at $620 \mathrm{~nm}$ was measured in both the forward and backward (towards the pump) directions, as backward emission contributed a significant fraction of the total power in this case. Output at the $3^{\text {rd }}$ Stokes in the backward direction was not measured because the dichroic mirror required to make the measurement reduced the incident pump power to below the threshold for the $3^{\text {rd }}$ Stokes.

Using this approach, a slope efficiency of $88 \%$ for the combined Raman output energy (black triangles and solid line in Figure 3a) was measured for the microlens cavity, equating to $84 \%$ pump to combined Raman conversion efficiency at the highest pump pulse energy of $16 \mu \mathrm{J}$, seen in Figure 3a. A reduced conversion efficiency of $59 \%$ was measured in the plane-plane case, with a slope efficiency of $74 \%$ (black triangles and solid line in Figure $3 \mathrm{~b}$ ). The maximum average powers of the combined Raman outputs were $134 \mathrm{~mW}$ and $96 \mathrm{~mW}$, respectively, for the micro-lens and plane-plane cases.

The Raman output in the microlens case had an $\mathrm{M}^{2}$ of $6.8 \times 4,1.9 \times 1.5$ and $1.5 \times 1.3$ for the $1^{\text {st }}, 2^{\text {nd }}$ and $3^{\text {rd }}$
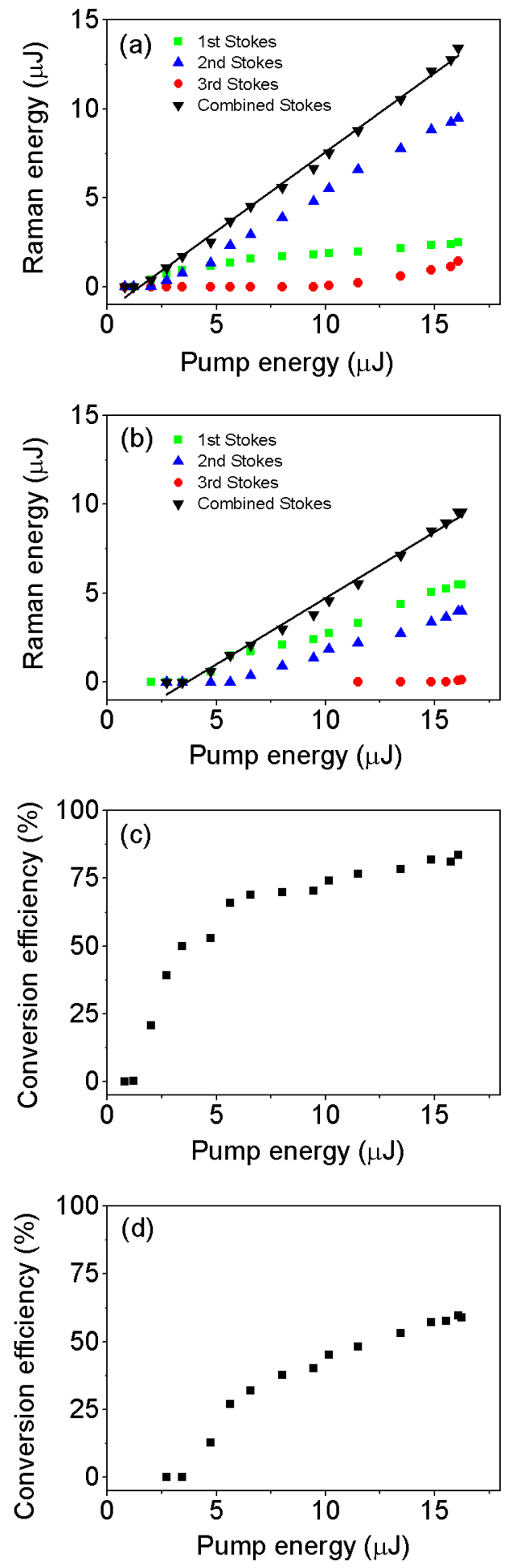

Figure 3. Energy transfer characteristics for the 1st (green squares) 2nd (blue triangles) and 3rd Stokes orders (red circles) and the combined Raman output (inverted black triangles) of (a) the microlens and (b) the plane-plane diamond Raman lasers, with (c) the conversion efficiency from pump to combined Raman in the microlens and (d) plane-plane cavities. 

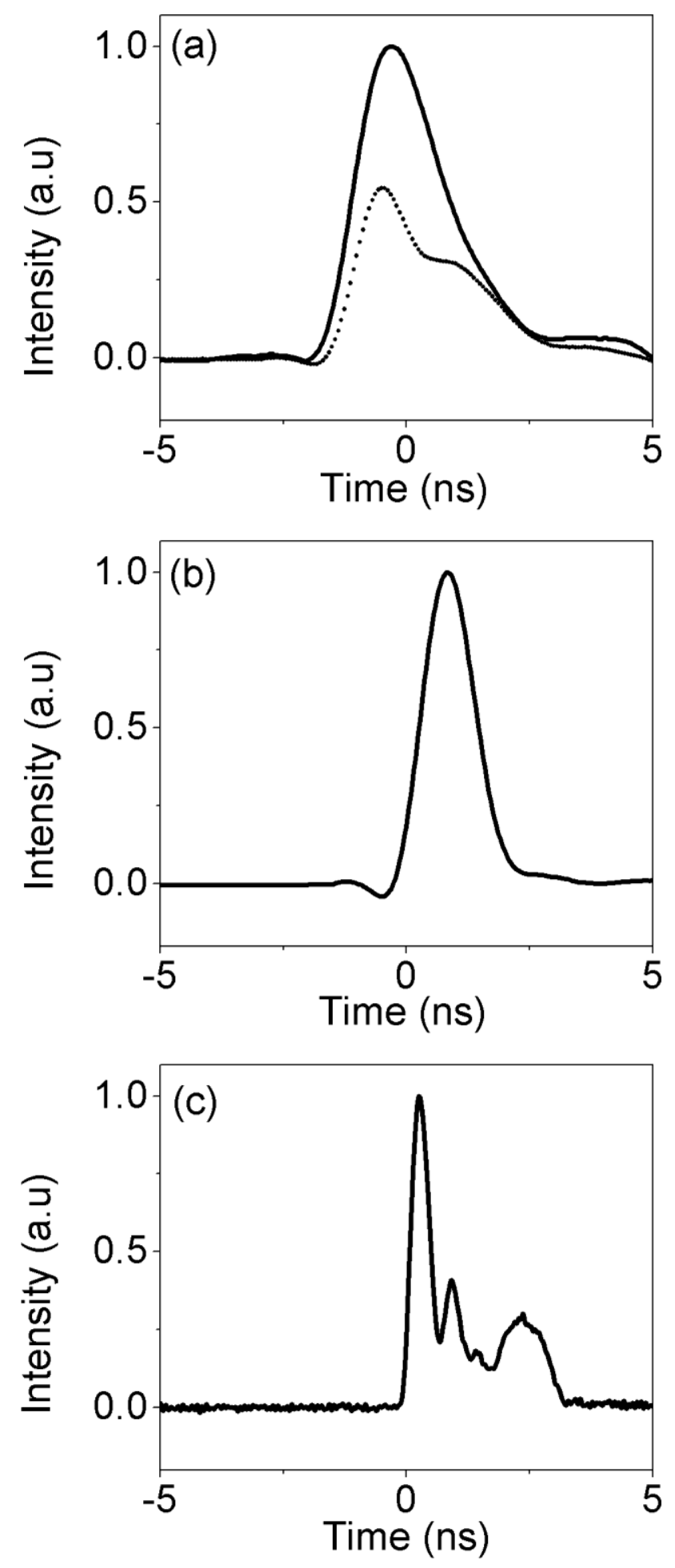

Figure 4. (a) Incident pump pulse (solid) compared with depleted pump pulse for the microlens case (dashed black), the measurement was taken at moderate pump powers. The $1^{\text {st }}$ Stokes pulse at 573nm in the microlens case: (b) below the second Stokes threshold, and (c) at maximum pump energy with both $2^{\text {nd }}$ and $3^{\text {rd }}$ Stokes oscillating.

Stokes, respectively, at maximum pulse energy. The $\mathrm{M}^{2}$ of the pump was $1.5 \times 1.4$. Issues with optical damage to the coatings made $\mathrm{M}^{2}$ measurements difficult for the planeplane case but values were no better than $3.6 \times 3,3.3 \times 2.8$ and $2.7 \times 2.7$ for the $1^{\text {st }}, 2^{\text {nd }}$ and $3^{\text {rd }}$ Stokes respectively.

The $1^{\text {st }}$ Stokes emission was polarized along a $<110>$ direction in the diamond for both the microlens and planeplane cavities, as would be expected from the polarization dependence of the Raman gain [3], [14]. No difference was observed in Raman output power when the microlens diamond was rotated for a pump polarization along a $<111>$ direction. Although, in theory, orientating the pump along a $<111>$ direction maximizes the Raman gain for propagation along <110> [3], [14], the Raman laser threshold is already small, so a significant change in Raman laser output power would not be expected.

Coating damage was observed much more readily on the plane-plane diamond than on the microlens crystal. Since the samples were coated in the same run, this might be indicative of poorer diamond surface quality in the plane-plane case.

The 1.5ns incident pump pulse is shown in Figure 4a (solid) along with the depleted pump (dashed). In the microlens and plane-plane cases, the $1^{\text {st }}$ Stokes pulse duration (FWHM) was 1.2ns at low pump energies (below the threshold of the $2^{\text {nd }}$ Stokes), as shown in Figure $4 \mathrm{~b}$. At maximum pump energy, however, depletion of the 1st Stokes by the 2nd Stokes, as shown in Figure 4c, meant the FWHM of the 1st Stokes pulse was not well defined. In the microlens case, the pulse duration of the $2^{\text {nd }}$ Stokes was measured to be $1.6 \mathrm{~ns}$ and the $3^{\text {rd }}$ to be $0.9 \mathrm{~ns}$. Pulse durations of $1.1 \mathrm{~ns}$ and $0.8 \mathrm{~ns}$ for the $2^{\text {nd }}$ and $3^{\text {rd }}$ Stokes respectively were measured for the plane-plane device. Due to the significant conversion from $2^{\text {nd }}$ to $3^{\text {rd }}$ Stokes in the microlens cavity, it appears depletion has artificially increased the FWHM of the $2^{\text {nd }}$ Stokes at maximum pump power.

The presence of three Stokes orders was confirmed using an Ocean Optics HR4000 spectrometer, with the spectrum shown in Figure 5. A spectrometer resolution of $0.13 \mathrm{~nm}$ meant any sub-structure in the peaks was unresolved.

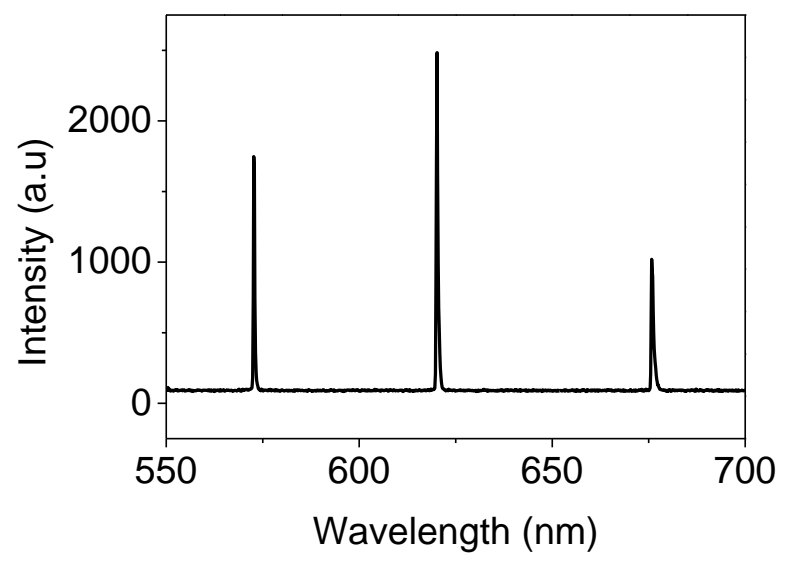

Figure 5. Typical spectral output in the microlens case showing the $1^{\text {st }}$ Stokes at $573 \mathrm{~nm}, 2^{\text {nd }}$ Stokes at $620 \mathrm{~nm}$ and $3^{\text {rd }}$ Stokes at $676 \mathrm{~nm}$. Similar spectra were obtained in the plane-plane case.

The observation of Raman laser oscillation in the planeplane case suggests that gain guiding [15] plays a role in transverse mode formation, since any thermal lens will be 
very weak. However, further experimental work is required to confirm this hypothesis and to elucidate any role gain-guiding might play in the microlens case.

A pronounced diffraction pattern is observed in the $1^{\text {st }}$ Stokes output beam from the plane-plane cavity at high pump energies (see Figure 6a). This may be the result of interplay between the transverse mode and the spatially varying pump depletion and hence gain. In the case of the microlens cavity, the diffraction pattern is less pronounced (see Figure 6b), perhaps due to the microlens structure stabilising the transverse mode. A detailed analysis of these effects will be the subject of future work.

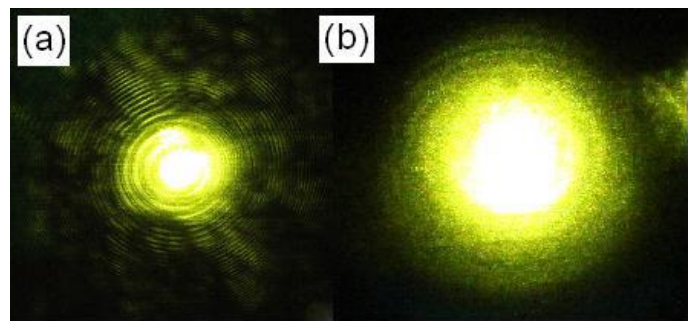

Figure 6. The $1^{\text {st }}$ Stokes output beam at maximum pump pulse energy from the plane-plane (a) and micro-lens (b) cavities. (Not to scale.)

This demonstration highlights the ease with which emission at the $1^{\text {st }}, 2^{\text {nd }}$ and $3^{\text {rd }}$ Stokes wavelengths can be generated in monolithic diamond Raman lasers. However, if only yellow output $(573 \mathrm{~nm})$ is desired, the use of coatings with greater transmission for the higher Stokes orders is likely to improve the conversion efficiency to the yellow by eliminating cascaded Raman conversion. This may also improve the output beam quality at the $1^{\text {st }}$ Stokes wavelength, which is currently being deteriorated by generation of higher Stokes components.

In conclusion, the feasibility of compact and robust monolithic diamond Raman lasers has been experimentally demonstrated. A conversion efficiency of $84 \%$ was achieved using microlens structures etched onto the front surface to form a stable cavity. In a simpler, plane-plane design, the Raman conversion efficiency was still 59\%. Multiple Stokes orders were observed, and with further optimization of optical coatings, highly efficient laser action at any one of these wavelengths should be attainable.

The authors gratefully acknowledge funding from the European Research Council (DiaL: 278389), the UK Engineering and Physical Sciences Research Council (EPSRC; EP/I029141/1) and via a Fraunhofer UK / Royal Academy of Engineering Research Chair (AJK). SR and HL acknowledge an EPSRC DTA studentship and a China Scholarship Council studentship, respectively.

\section{References}

[1] I. Friel, S. L. Geoghegan, D. J. Twitchen, and G. A. Scarsbrook, Proc. SPIE, 7838, 783819-1, (2010).

[2] V. G. Savitski, S. Reilly, and A. J. Kemp, IEEE J. Quantum Electron., 49, 218, (2013).

[3] V. G. Savitski, I. Friel, J. E. Hastie, M. D. Dawson, D. Burns, and A. J. Kemp, IEEE J. Quantum Electron , 48, $328,(2012)$.
[4] O. Kitzler, A. McKay, and R. P. Mildren, Opt. Lett., 37, 2790, (2012).

[5] A. McKay, H. Liu, O. Kitzler, and R. P. Mildren, Laser Phys. Lett., 10, 105801, (2013).

[6] E. Granados, D. J. Spence, and R. P. Mildren, Opt. Express, 19, 10857 (2011).

[7] J.-P. M. Feve, K. E. Shortoff, M. J. Bohn, and J. K. Brasseur, Opt. Express, 19, 913 (2011).

[8] A. Sabella, J. a Piper, and R. P. Mildren, Opt. Lett, 39, 4037 (2014).

[9] D. C. Parrotta, A. J. Kemp, M. D. Dawson, and J. E. Hastie, Opt. Express, 19, 24165 (2011).

[10] F. A. L'Espernace, Tr. Am. Opth. Soc, LXXXII, 82 (1985).

[11] J. R. F. De Abreu, International Opthalmology, 12, 193 (1988).

[12] R. P. Mildren and A. Sabella, Opt. Lett., 34, 2811 (2009).

[13] H. W. Choi, E. Gu, C. Liu, C. Griffin, J. M. Girkin, I.

M. Watson, and M. D. Dawson, J. Vac. Sci. Technol. B Microelectron. Nanom. Struct., 23, 130 (2005).

[14] A. Sabella, J. A. Piper, and R. P. Mildren, Opt. Lett., 35, 3874, (2010).

[15] F. Salin and J. Squier, Opt. Lett. 17, 1352 (1992). 
[12] R. P. Mildren and A. Sabella, "Highly efficient diamond Raman laser," Opt. Lett., vol. 34, no. 18, pp. 2811-2813, Sep. 2009.

[1] I. Friel, S. L. Geoghegan, D. J. Twitchen, and G. A. Scarsbrook, "Development of high quality single crystal diamond for novel laser applications," Proc. SPIE, vol. 7838, pp783819-1 - 783819-8, 2010.

[2] V. G. Savitski, S. Reilly, and A. J. Kemp, "Steady" State Raman Gain in Diamond as a Function of Pump Wavelength," IEEE J. Quantum Electron., vol. 49, no. 2, pp. 218-223, Feb. 2013.

[3] V. G. Savitski, I. Friel, J. E. Hastie, M. D. Dawson, D. Burns, and A. J. Kemp, "Characterization of Single-Crystal Synthetic Diamond for Multi-Watt Continuous-Wave Raman Lasers," IEEE JQE., vol. 48, no. 3, pp. 328-337, Mar. 2012.

[4] O. Kitzler, A. McKay, and R. P. Mildren, "Continuous-wave wavelength conversion for highpower applications using an external cavity diamond Raman laser," Opt. Lett., vol. 37, no. 14, pp. 2790-2792, Jul. 2012.

[5] A. McKay, H. Liu, O. Kitzler, and R. P. Mildren, "An efficient 14.5 W diamond Raman laser at high pulse repetition rate with first $(1240 \mathrm{~nm})$ and second (1485 nm) Stokes output," Laser Phys. Lett., vol. 10, no. 10, p. 105801, Oct. 2013.

[6] E. Granados, D. J. Spence, and R. P. Mildren, "Deep ultraviolet diamond Raman laser," Opt. Express, vol. 19, no. 11, pp. 10857-10863, May 2011.

[7] J.-P. M. Feve, K. E. Shortoff, M. J. Bohn, and J. K. Brasseur, "High average power diamond Raman laser," Opt. Express, vol. 19, no. 2, pp. 913-922, Jan. 2011.

[8] A. Sabella, J. a Piper, and R. P. Mildren, "Diamond Raman laser with continuously tunable output from 3.38 to $3.80 \mu \mathrm{m}$.," Opt. Lett., vol. 39, no. 13, pp. 4037-40, Jul. 2014.

[9] D. C. Parrotta, A. J. Kemp, M. D. Dawson, and J. E. Hastie, "Tunable continuous-wave diamond Raman laser," Opt. Express, vol. 19, no. 24, pp. 24165-24170, Nov. 2011.

[10] F. A. L’Espernace, "Trans-spectral Organic Dye Laser photocoagulation", Tr. Am. Opth. Soc, vol. LXXXIII, 82, 1985.

[11] J. R. F. De Abreu, "The tunable dye laser in the management of retinal vascular disease", International Opthalmology, vol. 196, pp. 193-196, 1988.

[13] H. W. Choi, E. Gu, C. Liu, C. Griffin, J. M. Girkin, I. M. Watson, and M. D. Dawson, "Fabrication of natural diamond microlenses by plasma etching," J. Vac. Sci. Technol. B Microelectron. Nanom. Struct., vol. 23, no. 1, pp. 130, 2005.

[14] A. Sabella, J. A. Piper, and R. P. Mildren, "1240 nm diamond Raman laser operating near the quantum limit," Opt. Lett., vol. 35, no. 23, pp. 3874-3876, Dec. 2010.

[15] F. Salin and J. Squier, "Gain guiding in solid-state lasers", Opt. Lett, vol. 17, no. 19, pp. 1352-1354, 1992. 